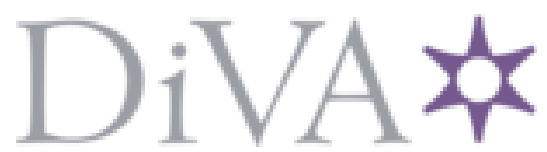

http://www.diva-portal.org

This is the published version of a paper presented at 2019 IST-Africa Week Conference (ISTAfrica.

Citation for the original published paper:

Kelati, A., DHAOU, I B., Kondoro, A., Rwegasira, D., Tenhunen, H. (2019)

IoT based Appliances Identification Techniques with FogComputing for e-Health In: 2019 IST-Africa Week Conference (IST-Africa Narobi, Kenya: IEEE https://doi.org/10.23919/ISTAFRICA.2019.8764818

N.B. When citing this work, cite the original published paper.

Permanent link to this version:

http://urn.kb.se/resolve?urn=urn:nbn:se:kth:diva-241293 


\title{
IoT based Appliances Identification Techniques with Fog Computing for e-Health
}

\author{
Amleset KELATI ${ }^{1,2}$, Imed BEN DHAOU ${ }^{4,5}$, Aron KONDORO ${ }^{1,3}$, Diana RWEGASIRA ${ }^{1,3}$, \\ Hannu TENHUNEN ${ }^{1,2}$ \\ ${ }^{1}$ KTH Royal Institute of Technology, Sweden \\ ${ }^{2}$ University of Turku, Finland \\ ${ }^{3}$ University of Dar es Salaam, Tanzania \\ ${ }^{4}$ Unaizah College of Engineering, Qassim University, Saudi Arabia \\ ${ }^{5}$ University of Monastir, Tunisia
}

Email:smleset@kth.se,phd.imed.benhaou@ieee.org,kondoro@kth.se,dianasr@kth.se,hannu@kth.se

\begin{abstract}
To improve the living standard of urban communities and to render the healthcare services sustainable and efficient, e-health system is experiencing a paradigm shift. Patients with cognitive discrepancies can be monitored and observed through the analyses of power consumption of home appliances. This paper surveys recent trends in home-based e-health services using metered energy consumption data. It also analyses and summarizes the constant impedance, constant current and constant power (ZIP) approaches for load modelling. The analysis briefly recaptures both non-intrusive and intrusive techniques. The work reports an architecture using IoT technologies for the design of a smart-meter, and fog-computing paradigm for raw processing of energy dataset. Finally, the paper describes the implementation platform based on GirdLAB-D simulation to construct accurate models of household appliances and test the machine-learning algorithm for the detection of abnormal behaviour.
\end{abstract}

Keywords: e-health., home management system, Internet of Things (IoT), fogcomputing, non-intrusive load monitoring and identification (NILM), smart-meter.

\section{Introduction}

Currently, $55 \%$ of the population of the world live in the main cities [1]; however, by 2050 the proportion is expected to reach $68 \%$. Additionally, as reported by the United Nations Population Fund, UNPF, the number of elderly people will rise to 2 billion in 2050[2]. Seniors tend to live independently and without direct interaction with assistance. However, caregivers need to monitor the health of the elderly people. The fact that seniors have a tendency of falling and are prone to physical injury, their lives are in constant. Those facts called for a paradigm shift in designing sustainable and affordable health services.

A smart city is an emerging concept that aims at integrating a plethora of advanced and smart services for the communities. The pillars of the smart-city model along with the number of indicators are described in Fig. [3]. Smart-living incorporates factors such as health, safety, housing, tourism, and culture.

Smart-home technologies allow for the integration and development of advanced health services through the daily monitoring of the activities of the end-user. Selection of sensors in the design of smart homes is one of the challenges as discussed in [4]. The fast communication between high- and low-level nodes of sensors make smart home a vibrant solution for monitoring the health of the occupant. Power consumption and the operation mode of the household appliances provide meaningful information for determining and 
predicting of the health and cognitive status of the user. Activity of daily living, ADL, is the best approach for determining the wellness of the person. Numerous published reports have proposed an ADL system for e-health, e.g., [5][6], and [7].

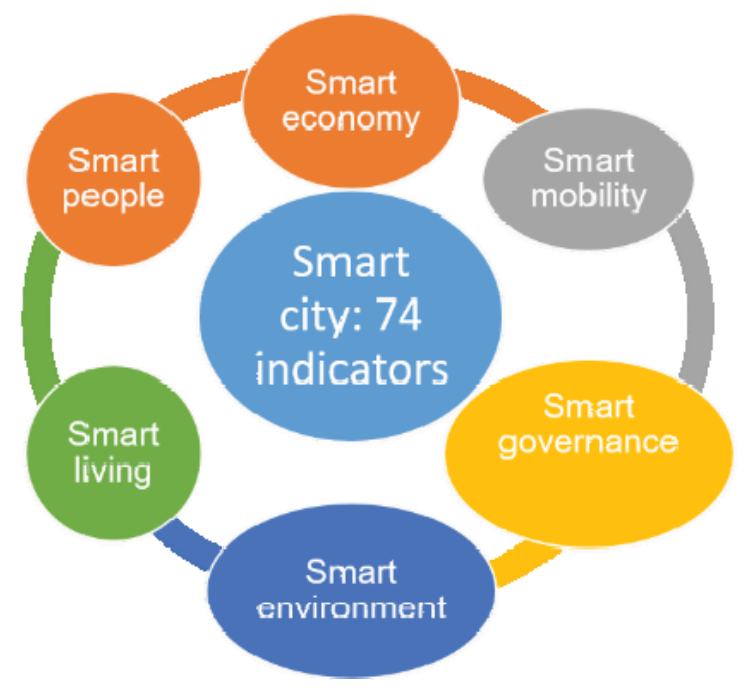

Figure 1: EU Model for the Smart City

Home management system (HEMS) has been the focus of some research work over the last decade [8]. The aim of HEMS is to lower the electricity bill and reduce the peak power consumption. The integration of HEMS with the e-health is an understudied subject where the aim is to develop algorithm and platforms that can be used to determine the health of the end-user [9]. Home integrated health monitoring (HIHM) is a real-time platform elaborated in [10] that measures, store and process vital health parameter of the patient.

Smart home-based health monitoring is an active research area where IoT based technologies are used to improve and maintain the healthcare of elderly and sick people. One of the key things that IoT has enabled has been the provision of critical contextual information in the healthcare process. The contextual information is vital since it provides understanding of the patient's environment, and improves the efficiency of the health monitoring process. This information includes data about the location, time, identity, and environment of the patient's surrounding. The load profiling methods on smart meter energy consumption data can be applied for the human behavioural model. Smart meter technology is a corner stone for monitoring, predicting, and controlling the energy consumption of the appliances. Collecting information on the daily activity of the seniors at home using smart meter provide a cost-efficient solution for e-health [11] [12] [13]. Here, the smart meter dataset information includes consumer's energy consumptions, the appliances usage information such as duration of appliances usage is an important factor for remote monitoring e-health system by promoting the independent living of seniors or patients with self-limiting health status.

There are a wide variety of smart home-based health monitoring systems that have been proposed in the literature [14]. In general, each of these systems has three main components: sensors, communication, and processing. Sensors are the devices that physically interact with the patient's environment and collect relevant health information to be used for decision-making. There are strong research efforts in both academia and private sector to develop new sensor devices that can be worn or embedded into patient's bodies and collect information that is more accurate. The communication component is the whole infrastructure that allows collected data to be transferred and stored for further processing. Different communication protocols for Home Area Networks (HANs) are being proposed to facilitate robust and efficient transport of vital health information. 
A non-intrusive load monitoring and identification (NILM) system is a technique, which has been widely used for appliance recognition and load forecasting. NILM can be used to collect ADL of the occupant. This work surveys recently published algorithms on nonintrusive load monitoring and describes an architecture for earlier detection of dementia and abnormal behavior. The rest of the paper is organized as follows. Section 2, describes load modelling approaches using non-intrusive load monitoring. Section 3 states the architecture. Section 4, surveys algorithms for appliances recognition and monitoring. The architecture of the e-health system described in Section 4. The implementation platform is discussed in Section 5. Section 6 describes the future research directions and concludes this work.

\section{Load Modelling}

The equivalent circuit model for the determination of AC appliances is depicted in Figure 2, where $\boldsymbol{Z}_{k}=Z_{k} \angle \theta_{k}$ is the impedance of the kth appliance.

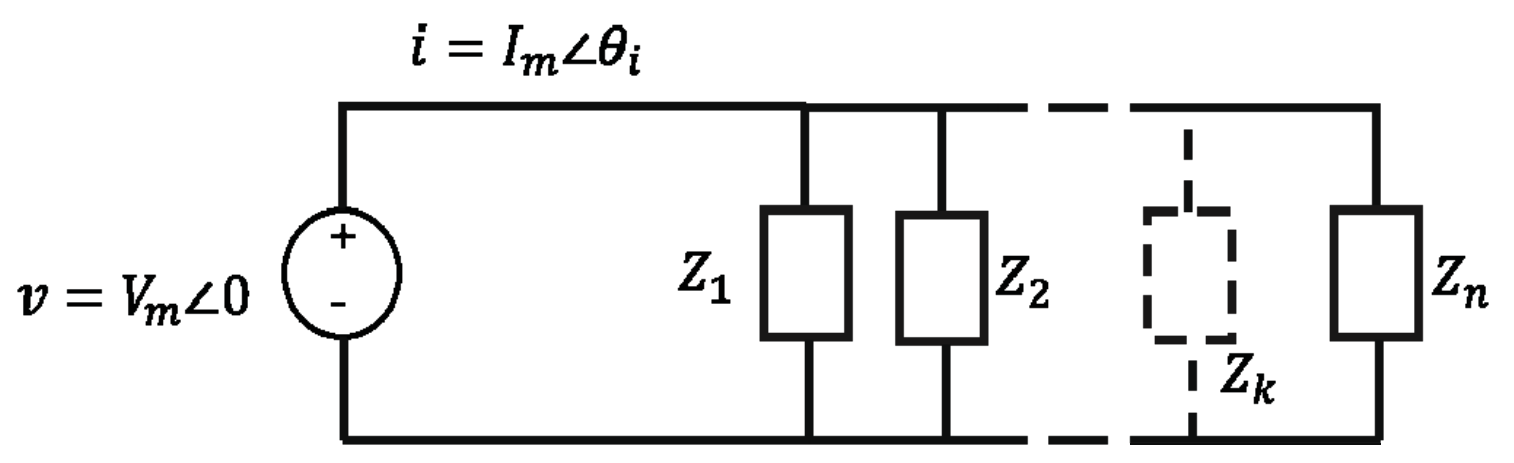

Figure 2: Circuit model of AC appliances in a given house

Let $\boldsymbol{Z}$ be the equivalent impedance of the circuit show above. The complex power consumption (apparent power) is given by (1).

$S=\frac{V_{m}^{2} \angle \theta}{z \angle \theta_{\pi}}=P+l Q,(1)$

Where $P$ is the average power consumption and $Q$ is the reactive power consumption. The determination of the complex power consumption is constrained with finding the appropriate impedance model of the appliance. In the literature, AC appliances have been grouped in three classes: predominantly resistive, predominantly inductive and predominantly capacitive [15].

The constant impedance, constant current, and constant power (ZIP) have been widely used to model AC appliances [16]. The ZIP model is referred to as the polynomial model, and it is static. The core methods for determining ZIP coefficients are shown in (2) and (3).

$$
\begin{aligned}
& P=P_{0}\left[Z_{P}\left(\frac{F}{V_{v}}\right)^{2}+I_{p} \frac{V}{V_{Q}}+P_{p}\right](2) \\
& Q=Q_{0}\left[Z_{Q}\left(\frac{F}{V_{0}}\right)^{2}+I_{Q} \frac{F}{V_{Q}}+P_{Q}\right](3)
\end{aligned}
$$

Where $P_{0}$ and $Q_{0}$ are, respectively, the rated active and reactive power. The ZIP coefficients for the active power $\operatorname{are} Z_{p}, I_{p}$, and $P_{p}$. The rest are the ZIP coefficients for the reactive power [17].

GridLAB-D, a defacto and open source simulation tool for power system developed by the US department of energy, uses ZIP load models. The ZIP equations for the active and reactive power are given in, respectively, (3) and (4).

$$
\begin{aligned}
& P=\frac{\psi^{0}}{V_{\theta}^{\theta}} Q_{0} Z \cos \left(Z_{\theta}\right)+\frac{\psi}{W_{0}} Q_{0} I \cos \left(I_{\theta}\right)+Q_{0} F \cos \left(F_{\theta}\right) \\
& P=\frac{V^{\theta}}{V_{\theta}^{\theta}} Q_{0} Z \sin \left(Z_{\theta}\right)+\frac{W}{W_{0}} Q_{0} I \sin \left(I_{\theta}\right)+Q_{0} P \sin \left(P_{\theta}\right)
\end{aligned}
$$


Where $Z_{\theta}$ is the phase angle of constant impedance fraction, $I_{\theta}$ is the phase angle of constant current fraction, $P_{\theta}$ is the phase angle of constant power fraction, $Z$ is the percent of load that is constant impedance, $P$ is the percent of load that is constant power, and $I$ is the percent of load that is constant impedance.

The ZIP models are static and hide paramount information that can be used to determine both the active and reactive power of home appliances. To address the shortcomings of the ZIP models a multi-state approach can be used to model the end-user load as advocated in [18]. The transition between states renders the system dynamic, in which a state can be modeled using ZIP or physical model.

In order to represent accurately the load characteristics of end user appliances, there have been attempts of developing new load models for modern equipment that appear in residential and industrial settings. These attempts fall under two categories: measurementbased and component-based. The measurement-based approach involves a direct collection of power measurement from loads that are subjected to varying voltage conditions. The component-based approach involves the analysis of components that constitute a load to derive the model that describes it. Both categories of approaches lead to a ZIP model that estimates the power consumption of electrical appliances under different voltage conditions. [19] develop new load models for new types of customer equipment using a component-based approach. Several types of loads were tested in a lab environment under varying voltage conditions. Power was obtained from the grid and the voltage was varied using a variable autotransformer. The research develops a constant impedance-currentpower (ZIP) model for these new devices. The new models facilitate the more accurate estimation of load profiles of customers with these devices under different voltage conditions. The component-based approach is also used to develop new load models for residential and industrial equipment [20]. They tested a variety of modern household's appliances and industrial equipment to develop ZIP models that describe their steady-state behavior. Based on a survey conducted in homes and businesses available in New York City, different types of loads that accurately reflect reality was collected and assembled in a lab environment. These devices were analyzed and categorized into different classes depending on their operational characteristics. They were subjected to different voltage conditions in a lab environment and power measurements were collected. Derived models were then validated against actual measurements of loads in the power system.

Load signature approach has been proposed as an alternative to the ZIP model. The chief idea for load signature is to determine the feature of an AC appliance. A comprehensive review on this topic is reported in [41]. Signatures can be determined at the micro-level (sampling rate less than 1 sample/ cycle) and macro-level (sampling rate higher than 1 sample/ sec) [39]. Traditionally, active and reactive power are used to determine the load signature [21], the authors of [Signature1] added six more signatures: eigen value, current waveform, harmonics, instantaneous power waveform, instantaneous admittance waveform, and switching transient waveform. A total of 27 home appliances have been characterized using the seven features. Although, accurate, these seven models are computationally heavy and cannot be used for real-time monitoring. Additionally, the current waveform $(\mathrm{CW})$ is not unique for some appliances. To address these shortcomings, $\mathrm{CW}$ has to be decomposed into active and non-active current [40].

In summary, three techniques are widely considered for appliances modelling. These techniques are: ON/OFF, finite state machine (FSM), and continuously variable.

Table 1summarize the appropriateness of each model [21]. 
Table 1: Appliances Modeling

\begin{tabular}{|l|l|}
\hline Model & Sample Appliances \\
\hline ON/OFF & $\begin{array}{l}\text { Toaster, light bulb, water pump, coffee } \\
\text { machines, etc. }\end{array}$ \\
\hline FSM & $\begin{array}{l}\text { Processor-controlled appliances, washing } \\
\text { machines, dishwasher, refrigerator, etc. }\end{array}$ \\
\hline Continuously variable & $\begin{array}{l}\text { Light dimmers, variable-speed drive, heat } \\
\text { pumps, etc. }\end{array}$ \\
\hline
\end{tabular}

\section{Algorithms for Appliances Identification and Monitoring}

In the past, non-intrusive load monitoring, NILM, has been used for load forecasting, price regulations, appliance designs, etc., [21]. Both manual and automatic NILMs have been used. The former requires a setup period in which a lookup table is created to recognize the signature of the appliance that latter automatically builds the signature table and needs priory information such as type and name of the appliance.

The NILM records the appliances usage time and usage frequency [11]. The assumption was to detect a behavioral change using electric load intelligence [39].

Detecting human activities and sudden changes can be utilized for the health provider's attention[22]. The activity prediction depends on the analysis of the usage of multiple appliances with the Bayesian network. The time series based multi-label classifier has been used for the prediction of the home energy usage using appliances at homes [23] to get results with high-level accuracy. The approach taken was the k-means for clustering algorithms using the pattern analysis that related to sudden changes and the Bayesian networks for activity prediction related to health activities based on historical data.

A non-intrusive load monitoring and identification, NILM, system is used for investigating the electricity usage of the residents [24]. The machine learning method can analyze and classify appliances with a unique signature. It can distinguish anonymous appliances and arrange relevant actions to enhance the classification accuracy. Machine learning algorithm strategy can identify appliances with an arbitrary accuracy if the power is measured every $10 \mathrm{sec}$ or less [25]. The same approach was used by [26] in India to come up with four feature which is important. These are load signatures of active and reactive powers, harmonic components and their magnitudes. The algorithms have three assumptions: single-phase appliances, the algorithm is based strictly on the specifications of IEW (Internal Electrical Wiring) and depend on steady-state values of features and Table 3 summarize the signature-based approaches for appliances identification.

Table 2: Load Monitoring Techniques are Categorized with Two Main Methods

\begin{tabular}{|l|l|l|}
\hline Method & Description & Method \\
\hline $\begin{array}{l}\text { Intrusive Load } \\
\text { (ILM) }\end{array}$ & $\begin{array}{l}\text { The measurement is taking } \\
\text { inside the house by connecting } \\
\text { each appliance physically to the } \\
\text { measurement device and are } \\
\text { connected to a hub or smart } \\
\text { meter. The method has an } \\
\text { accurate measurement but } \\
\text { expensive } \\
{[27] .}\end{array}$ & $\begin{array}{l}\text { Supervisory Control and data } \\
\text { acquisition (SCADA). } \\
\text { Identification is done with an } \\
\text { algorithm that help to } \\
\text { distinguish each appliances a } \\
\text { known feature energy } \\
\text { consumption. Direct or indirect } \\
\text { monitoring [27] [28], based on } \\
\text { power demand [29]. of a typical } \\
\text { appliance from the data }\end{array}$ \\
\hline $\begin{array}{l}\text { Non- intrusive } \\
\text { Load }\end{array}$ & $\begin{array}{l}\text { Each appliance the operation } \\
\text { state and energy consumption is }\end{array}$ & $\begin{array}{l}\text { Appliances identification is by } \\
\text { monitoring the change in active }\end{array}$ \\
\hline
\end{tabular}




\begin{tabular}{|l|l|l|}
\hline $\begin{array}{l}\text { Monitoring } \\
\text { (NILM) }\end{array}$ & $\begin{array}{l}\text { depend on the power } \\
\text { measurement of the aggregate } \\
\text { load of the building. [30], [31] } \\
{[32] .}\end{array}$ & $\begin{array}{l}\text { and reactive power and by } \\
\text { setting the operating state of the } \\
\text { appliances. Hidden Markov } \\
\text { Model (HMM) [33] [35], } \\
\text { identification by switch-on and } \\
\text { off the appliances and changes } \\
\text { in the pre-processed from } \\
\text { steady-state or transient to detect } \\
\text { the appliances [34]. }\end{array}$ \\
\hline
\end{tabular}

Table 3. Methods for Signatures on Appliances Identification

\begin{tabular}{|l|l|}
\hline Signatures & Description \\
\hline Energy Disaggregation & $\begin{array}{l}\text { Depend on the individual appliance in the total load [32], } \\
\text { switch ON and OFF power change in steady and the } \\
\text { event is the basis for frequency or harmonic frequency } \\
\text { signatures [30]. }\end{array}$ \\
\hline Fundamental Frequency & $\begin{array}{l}\text { Measuring current, power, or the normalized admittance } \\
\text { power is the change as signatures [30]. }\end{array}$ \\
\hline Harmonic frequency & $\begin{array}{l}\text { Appliances produce harmonic current and for some } \\
\text { appliances not easy to distinguish using only reactive or } \\
\text { real power the signature is based on transient and steady } \\
\text { state [35]. }\end{array}$ \\
\hline Steady-State Signatures & $\begin{array}{l}\text { Connected to the states on the operating level [30]. } \\
\text { reactive and active and power and current } \\
\text { waveform, include temporal motif mining } \\
\text { approach [36], combined HMM load model [37] } \\
\text { Current waveform characteristics, Different } \\
\text { current measurements used for load identification } \\
\text { [35] and the method is often used on reactive and } \\
\text { real power and transients power [38]. }\end{array}$ \\
\hline
\end{tabular}

The energy management system is equipped to provide reliable monitoring and appliances identification. Smart grid technology enables consumers to know and manage their electrical loads and smart meter enable the communications between the electrical utility, energy demand, and pricing. Individual appliances load is measured with the connected plug load meters that are resalable for real-time energy management. The measures can identify the on /off state, the change in the state and the information on the peak power consumption of the appliances [34].

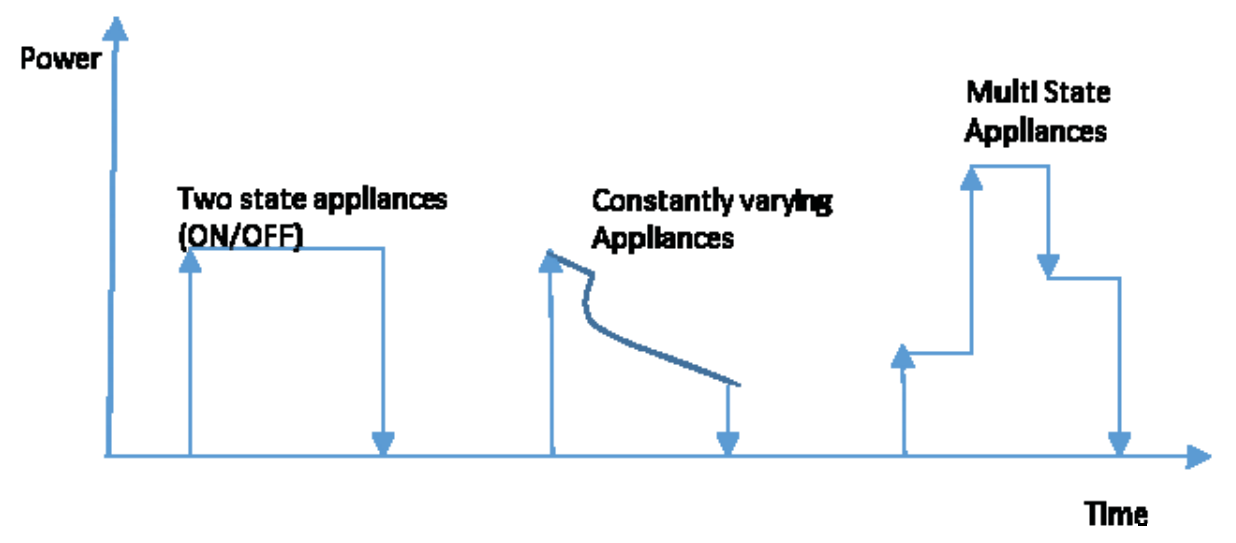

Figure 3. Different Appliances Types Based on their Energy Consumption Pattern [35]. 
The appliances power consumption can be measured with low frequency to obtain an accuracy of classifications. The measurements of the appliances power consumption can be described with the known basic state of mode as describe in the literature [25].

To consider accurate appliances identifications and classifications, the measured power consumption will undergo different steps: pre-processing, feature extraction, splitting, post-processing, and training \& classification. For stable and accurate appliance identification, considering the methodology of machine learning algorithms for processing will be beneficial. The process follows the procedure, which include collecting the readings of the appliances feature from the dataset and performing feature extraction to figure out and understand the power consumption dataset to be used for the appliances identifications.

The real-time classification of appliances is set according to energy consumption and can be used in diverse applications scenarios. One application is associated with human activity observation and detect abnormalities that related to health conditions. A smart meter is connected to the household for real-time load profiling of the power consumption of the appliances through the remote controlling to apply for several and different applications. The measurements and extraction of the power consumption pattern of each appliance have a unique pattern that depends on the data acquisition. The complicated behavior of the appliances acquires different activity and functionality and external circumstance influence the process of identification and label the appliances signature.

\section{System Architecture}

Load profiling and recognition necessities a constant learning process in order to improve efficiency and reduce the false alert ones. The system architecture as depicted in Figure 4, consists of the following entities. A smart plug through which a home appliance gets the power. The smart plug is an embedded system that houses sensors along with the communication and DSP processing blocks. The plug can also control the operation of the appliance to support the demand response scheme. The plugs within the house are connected through a various communication link to the smart meter. The smart meter aggregates the data from all plugs, process it and ferry it to a server for health-care diagnosis and other home energy management functions.

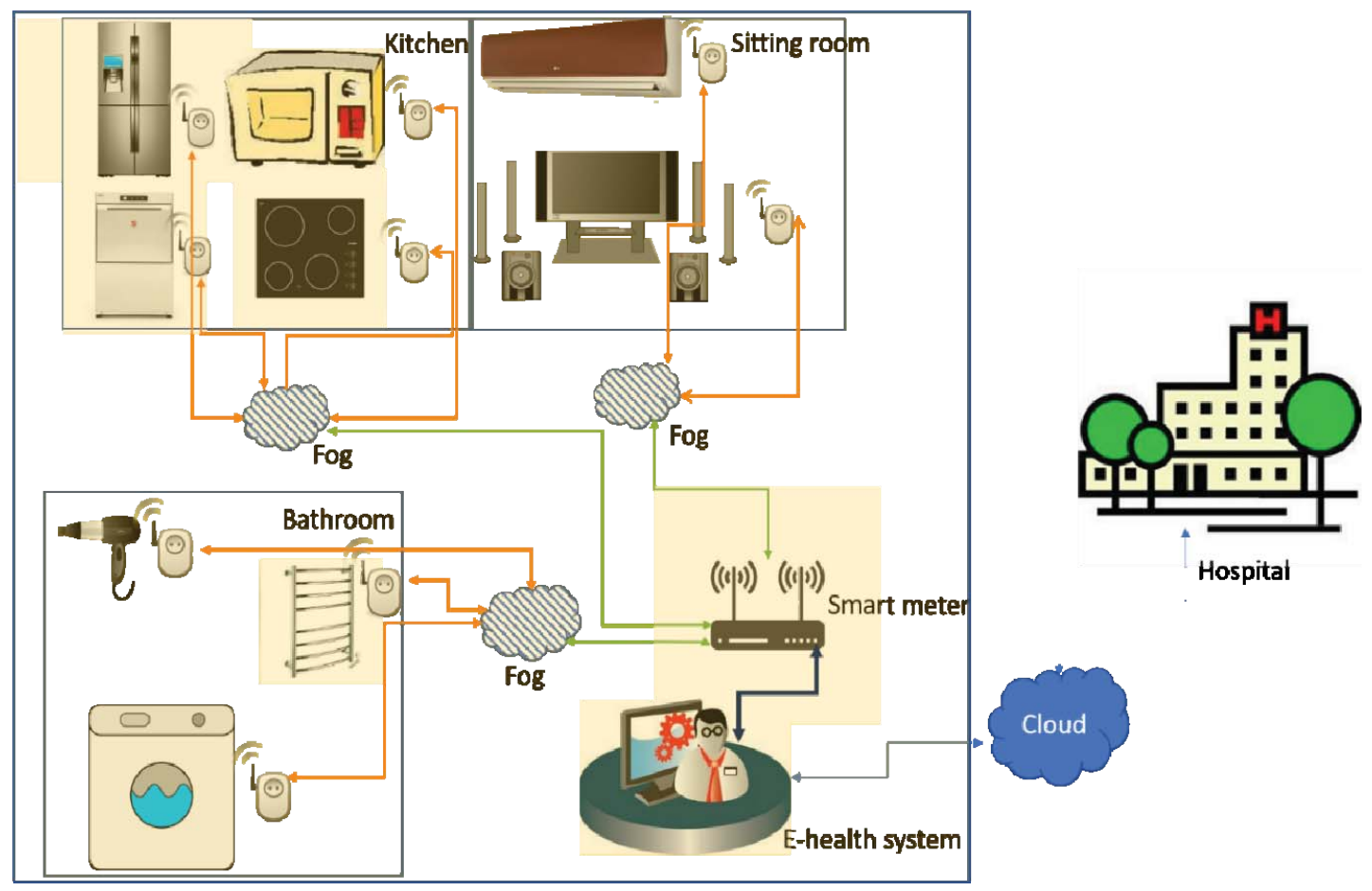

Figure 4. Smart Home Architecture 
New architectural designs based on fog computing that utilize existing communication devices in homes are also being proposed to improve performance and deployment. The processing system includes all backend devices and algorithms that enable the automation of health-based decisions. New architectural designs based on fog computing that utilize existing communication devices in homes are also being proposed to improve performance and deployment. The processing system includes all backend devices and algorithms that enable the automation of health-based decisions. New approaches based on big data and machine learning are being used to detect and predict health anomalies. Figure 5. shows, the three layers of fog hierarchies; the nodes at bottom layer gather the smart meter measurements from home appliances and facilitate an early warning that needs to detect the abnormal status immediately by communication to the cloud platform. The architecture is composed of the following three layers. The Smart gird layer has a smart meter and responsible for communicating the appliances and smart meter, and other smart grid devices. The Fog layer communicates smart meter measurements and data to the cloud by separating different types of user data. The cloud layer data can be stored for further analysis mainly on identification or some prediction and sent to Fog computing. It is possible the detailed and private data are normally kept in cloud servers.

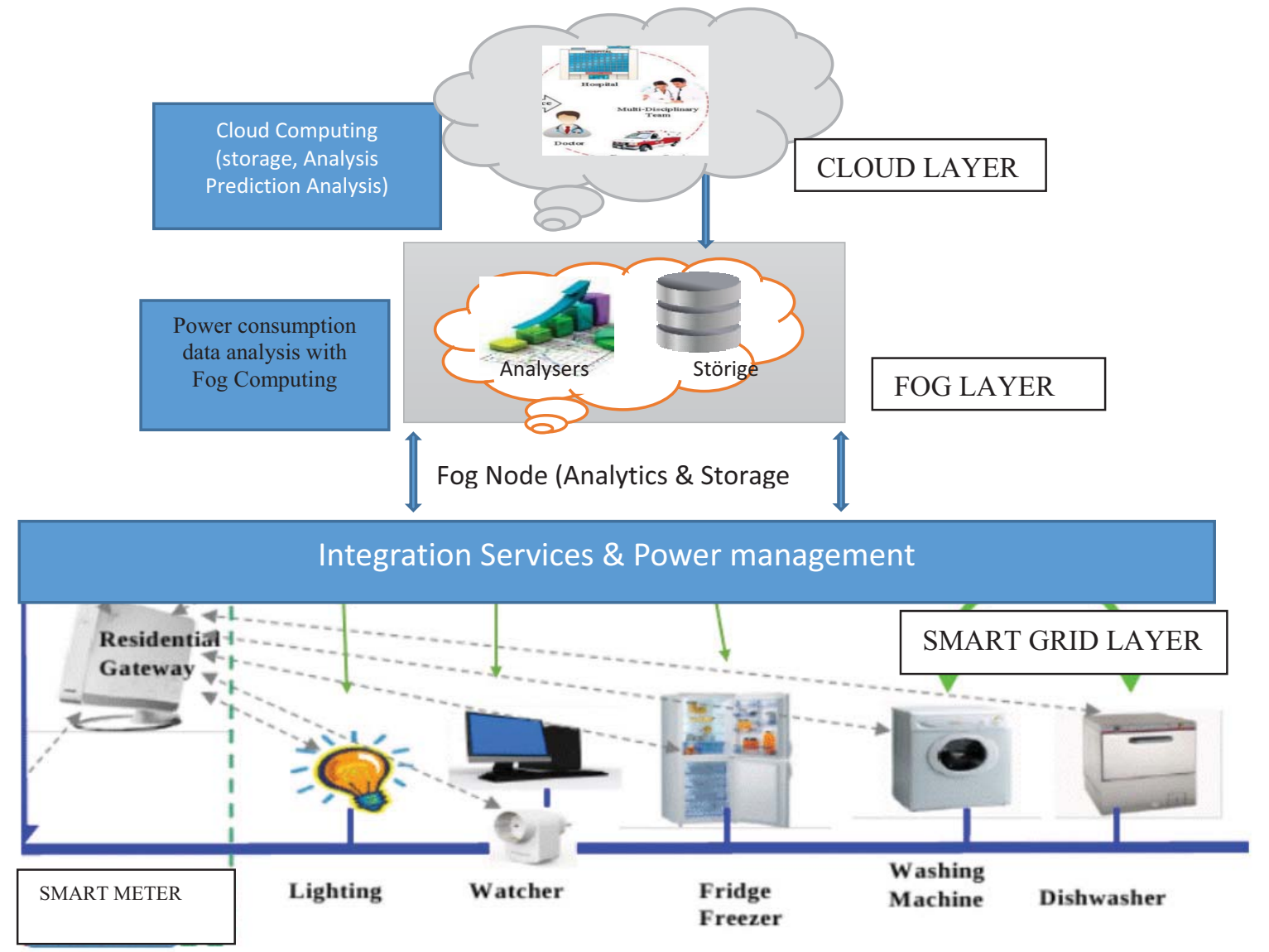

Figure 5. Energy Consumption Analysis in Fog / Cloud Computing

\section{Implementation Platform}

There exist multiple candidates (hardware and software) for the implementation of the home-based e-health system. First, the power consumption dataset of the appliance gathered by using wireless sensor networks. At this stage the communication protocol is on the Smart-plug with an IoT can be used at this level. The purpose lies on the detecting the behavioural changes on the appliances usage of the consumers at home in the case of the appliances regularly monitored as they are used by the occupants in the house. The activity 
with the interaction of the appliances is stored in the computer or laptop in order to determine the status of health in our case for instance for elderly people. The information from the sensor and smart plug is equipped with how, when or for how long the interaction and activity of the appliances. The data processing part of the smart-plug is accomplished with the help of a microcontroller such as Arduino. The smart meter is the device that gathers data from the smart plug. There are multiple platforms for implementation of the smart meter. To cope with the heterogeneity of communication protocols, IoT middlewares are received an ample interest for the realization of the smart-meter devices. The smart meter measurement data describe timely dependant load profiling for load forecasting and apply the NILM method for household appliances from consumers.

To test the NILM for e-health the implemetation is done with GridLAB-D which is a promising simulation platform that has abundant accurate models of the home appliances. The features of GridLAB-D have a user friendly and the open source code that is available for edit as needed. The platform has a multi-state time variant scheduling load models that are flexible load profiling simulation related to the end user appliances voltage. The platform can run on multiprocessor machine and use multi-agent techniques and allows deploying new control algorithms. Our implementation for load modelling is described briefly in section 2. The implementation uses the GridLAB-D IEEE 13 node stranded for the current, voltage control and load flow of the and energy storage for the appliances measurement on its simulation.

Table 4 summarizes hardware and software tools to simulate the proposed prototype

Table 4: Implementation platforms

\begin{tabular}{|l|l|l|}
\hline Device/tool & Functions & Candidates \\
\hline Current sensors & $\begin{array}{l}\text { Measures the instantaneous } \\
\text { power consumption and } \\
\text { RMS value }\end{array}$ & ACS712 \\
\hline Microcontrollers & $\begin{array}{l}\text { Filtering, calculation of ZIP, } \\
\text { communication, appliance } \\
\text { identification, smart- } \\
\text { metering }\end{array}$ & $\begin{array}{l}\text { Atmel } \\
\text { AVR465, } \\
\text { MCF51EM256, } \\
\text { TMPM411F20 }\end{array}$ \\
\hline Middleware & Security, heterogeneity & \multicolumn{1}{|c|}{ KAA } \\
\hline Power system simulator & Simulation, modelling & $\begin{array}{c}\text { GridLAB-D and } \\
\text { Matlab }\end{array}$ \\
\hline
\end{tabular}

\section{Conclusion}

E-health using electric load intelligence is a promising technology for home-based healthcare system. The idea behind the system is to identify the type and operation mode of the electric appliance using aggregated energy measurement, then determine the health of the occupant using a predefined mode of operation. This paper has surveyed the latest published algorithms for appliances identifications. It also discussed the load modelling techniques. Dynamic ZIP model is the most suitable candidate for load modelling. The construction of the models needs a priori knowledge of the appliance to measure the significant parameters such as rated active power and reactive power, current, and impedance. Finally, the present work devised a fog-based architecture for the e-health system. Our future work will focus on devising an algorithm for appliances identification using machine-learning techniques and the algorithm implementation on FPGA to obtain efficient identification of hardware architecture. 


\section{Acknowledgement}

This work is funded by the Swedish Funding Agency, SIDA and supported through iGrid Project at the Division of Electronics, School of Electrical Engineering and Computer Science, Royal Institute of Technology (KTH).

\section{References}

1. United Nation. (2014). World Urbanization Prospect. [Online]. Available: https://www.un.org/development/desa/publications/2018-revision-of-world-urbanization-prospects.html.

2. N. K. Suryadevara, S. C. Mukhopadhyay, R. Wang, R. K. Rayudu and Y. M. Huang, "Reliable measurement of Wireless Sensor Network data for forecasting wellness of elderly at smart home," 2013 IEEE International Instrumentation and Measurement Technology Conference (I2MTC), Minneapolis, MN, 2013, pp. 16-21.

3. Rudolf Giffinger et al., "Smart cities Ranking of European medium-sized cities", Research Institute for Housing, Urban and Mobility Studies (OTB), October 2017, www.smart-cities.eu,http://www.smartities.eu/download/smart_cities_final_report.pdf.

4. V. Nathan et al., "A Survey on Smart Homes for Aging in Place: Toward Solutions to the Specific Needs of the Elderly," in IEEE Signal Processing Magazine, vol. 35, no. 5, pp. 111-119, Sept. 2018.

5. H. Pazhoumand-Dar, M. Masek and C. P. Lam, "Unsupervised monitoring of electrical devices for detecting deviations in daily routines," 2015 10th International Conference on Information, Communications and Signal Processing (ICICS), Singapore, 2015, pp. 1-6.

6. S. Rahimi, A. D. C. Chan and R. A. Goubran, "Usage monitoring of electrical devices in a smart home," 2011 Annual International Conference of the IEEE Engineering in Medicine and Biology Society, Boston, MA, 2011, pp. 5307-5310.

7. N. Noury, M. Berenguer, H. Teyssier, M. Bouzid and M. Giordani, "Building an Index of Activity of Inhabitants From Their Activity on the Residential Electrical Power Line," in IEEE Transactions on Information Technology in Biomedicine, vol. 15, no. 5, pp. 758-766, Sept. 2011.

8. N. Javaid, I. Khan, M. N. Ullah, A. Mahmood and M. U. Farooq, "A Survey of Home Energy Management Systems in Future Smart Grid Communications," 2013 Eighth International Conference on Broadband and Wireless Computing, Communication and Applications, Compiegne, 2013, pp. 459-464.

9. A. Yassine, S. Singh and A. Alamri, "Mining Human Activity Patterns From Smart Home Big Data for Health Care Applications," in IEEE Access, vol. 5, pp. 13131-13141, 2017.

10. J. S. Kim, B. O. Kim and K. S. Park, "Development of HIHM (Home Integrated Health Monitor) for Ubiquitous Home Healthcare," 2007 29th Annual International Conference of the IEEE Engineering in Medicine and Biology Society, Lyon, 2007, pp. 363-365.

11. J. Liao, L. Stankovic, and V. Stankovic, "Detecting household activity patterns from smart meter data," in Proc. Int. Conf. Intell. Environ. (IE), vol. 6. Jul. 2014, pp. 7178.

12. A. Yassine, A. A. Nazari Shirehjini and S. Shirmohammadi, "Smart Meters Big Data: Game Theoretic Model for Fair Data Sharing in Deregulated Smart Grids," in IEEE Access, vol. 3, pp. 2743-2754, 2015.

13. H. Ghayvat, S. Mukhopadhyay, B. Shenjie, A. Chouhan and W. Chen, "Smart home based ambient assisted living: Recognition of anomaly in the activity of daily living for an elderly living alone," 2018 IEEE International Instrumentation and Measurement Technology Conference (I2MTC), Houston, TX, 2018, pp. 1-5.

14. A survey on health monitoring systems for health smart homes, Haider Mshali a, Tayeb Lemlouma b, *, Maria Moloney c, Damien Magoni a, International Journal of Industrial Ergonomics, pp. 26-56.

15. A. Ridi, C. Gisler and J. Hennebert, "A Survey on Intrusive Load Monitoring for Appliance Recognition," 2014 22nd International Conference on Pattern Recognition, Stockholm, 2014, pp. 37023707.

16. Alcalá JM, Ureña J, Hernández Á, Gualda D. Assessing Human Activity in Elderly People Using NonIntrusive Load Monitoring. Sensors (Basel). 2017;17(2):351. Published 2017 Feb 11.

17. "Standard load models for power flow and dynamic performance simulation", IEEE Transactions on Power Systems, vol. 10, no. 3, pp. 1302-1313, 1995. Available: 10.1109/59.466523.

18. K. Schneider, J. Fuller and D. Chassin, "Multi-State Load Models for Distribution System Analysis", IEEE Transactions on Power Systems, vol. 26, no. 4, pp. 2425-2433, 2011. Available: 10.1109/tpwrs.2011.2132154.

19. F. L. Quilumba, W.-J. Lee, H. Huang, D. Y. Wang, and R. L. Szabados, "Load model development for next generation appliances," in 2011 IEEE Industry Applications Society Annual Meeting, 2011.

20. A. Bokhari et al., "Experimental Determination of the ZIP Coefficients for Modern Residential, Commercial, and Industrial Loads,” IEEE Trans. Power Delivery, vol. 29, no. 3, pp. 1372-1381, 2014. 
21. G. Hart, "Nonintrusive appliance load monitoring", Proceedings of the IEEE, vol. 80, no. 12, pp. 18701891, 1992. Available: 10.1109/5.192069.

22. A. Yassine, S. Singh and A. Alamri, "Mining Human Activity Patterns From Smart Home Big Data for Health Care Applications," in IEEE Access, vol. 5, pp. 13131-13141, 2017.

23. K. Basu, V. Debusschere and S. Bacha, "Appliance usage prediction using a time series based classification approach," IECON 2012 - 38th Annual Conference on IEEE Industrial Electronics Society, Montreal, QC, 2012, pp. 1217-1222.

24. Rossier, Florian, et al. "Near Real-Time Appliance Recognition Using Low-Frequency Monitoring and Active Learning Methods.” Energy Procedia, vol. 122, 2017, pp. 691-696.

25. Srinivasan Iyengar, David Irwin, and Prashant Shenoy. "Non-intrusive model derivation: automated modeling of residential electrical loads", In ACM e-Energy. 2016

26. Sunil Semwal, R S Prasad, Pradeep Juneja "Identifying Appliances using NIALM with Minimum Features", December 2014 International Journal of Electrical and Computer Engineering 4(6):909-922.

27. M. Zeifman, K. Roth, "Non-Intrusive load Monitoring: Review and outlook", Journal IEEE Transactions on Consumer Electronics, Volume 57 Issue 1, February 2011 , Page 76-84.

28. Yoshimoto, K., Nakano, Y., Amano, Y., and Kermanshahi, B.. "Non-Intrusive Appliances Load Monitoring System Using Neural Networks.", IEEE Proceedings of International Symposium on Circuits System, Vol. 2, pp.183-194.

29. Breed, J.A., and Delport, G.J. (2001). Non-Intrusive Load Monitoring of Residential Appliances. Proceeding of the 7th Conference on Domestic Use of Energy, pp.33-38.

30. Ehrhardt-Martinez, K. Donnelly, K.A. and John. A. (2010). Advanced Metering Initiatives and Residential Feedback Programs: A Meta-Review for Household Electricity-Saving Opportunities. Technical Report E105 for American Ccouncil for Energy Efficient

31. Zoha, A., Gluhak, A., Imran MA., and Rajasegarar, S. (2012). Non-Intrusive Load Monitoring Approaches for Disaggregated Energy Sensing: A Survey,” Open Access Sensor ISSN 1424-8220, pp.16838-16866.

32. Z. Wang and G. Zheng, "Residential Appliances Identification and Monitoring by a Nonintrusive Method," in IEEE Transactions on Smart Grid, vol. 3, no. 1, pp. 80-92, March 2012.

33. Phial, H. (1998). Non-Intrusive Appliance Load Monitoring Systems Based on a Modern kWh-Meter," VTT Energy, Thesis Submitted for Official Examination for The Degree of Licentiate in Technology, Espoo.

34. Barges, M.E., Goldman, E., Matthews, H.S., and Lucio Soibelman, L. "Enhancing Electricity Audits in Residential Buildings with Nonintrusive Load Monitoring." Journal of Industrial Ecology, Vol. 14, No.5, pp.1-15, Oct. 2010.

35. Y. Du, L. Du, B. Lu, R. Harley and T. Habetler, "A review of identification and monitoring methods for electric loads in commercial and residential buildings," 2010 IEEE Energy Conversion Congress and Exposition, Atlanta, GA, 2010, pp. 4527-4533.

36. Shao, H., Marwash, M. and Ramarkrishnam, N. (2012). A Temporal Motif Mining Approach to unsupervised Energy Disaggregation: Application to Residential and Commercial Buildings. 1st International Workshop on Non-Intrusive Load Monitoring, pp, 1-7.

37. Kolter, J. Z. and Jaakkola, T. (2012). Approximate Interface in Additive Factorial HMMs with Application to Energy Disaggregation," Proceeding of the Fifteenth Conference on Artificial Intelligence and Statistics, pp.1472-1482.

38. M. Ito, R. Uda, S. Ichimura, K. Tago, T. Hoshi and Y. Matsushita, "A method of appliance detection based on features of power waveform," 2004 International Symposium on Applications and the Internet. Proceedings., Tokyo, Japan, 2004, pp. 291-294.

39. J. Liang, S. K. K. Ng, G. Kendall and J. W. M. Cheng, "Load Signature Study_Part I: Basic Concept, Structure, and Methodology," in IEEE Transactions on Power Delivery, vol. 25, no. 2, pp. 551-560, April 2010.

40. T. D. Huang, W. Wang and K. Lian, "A New Power Signature for Nonintrusive Appliance Load Monitoring," in IEEE Transactions on Smart Grid, vol. 6, no. 4, pp. 1994-1995, July 2015.doi: 10.1109/TSG.2015.2415456.

41. N. Sadeghianpourhamami, J. Ruyssinck, D. Deschrijver, T. Dhaene, C. Develder, "Comprehensive feature selection for appliance classification in NILM", Energy and Buildings, vol. 151, pp. 98, 2017. 